\title{
O PAPEL DOS FATORES GENÉTICOS NA OTITE MÉDIA
}

\author{
THE ROLE OF GENETIC FACTORS IN OTITIS MEDIA
}

\author{
Luiz Lavinsky ${ }^{1}$, Andréa M. Campagnolo², Ana Paula G. Raupp², Ângela B. John², \\ Cláudia Helena G. Estrella², Cristina C. Comiran² \& Thêmis M. Félix ${ }^{3}$
}

\begin{abstract}
'Docente; ${ }^{2}$ Doutorandas - Faculdade de Medicina da Universidade Federal do Rio Grande do Sul; ${ }^{3}$ Médica do Serviço de Genética do Hospital das Clínicas de Porto Alegre.

CorResPondÊnCIA: Dr. Luiz Lavinsky - Clínica Lavinsky - Rua: Quintino Bocaiúva, 673; CEP 90440-051 - Porto Alegre, RS - Brasil. Fone: (051) 330-2444 - Fax: (051) 330-6834
\end{abstract}

LAVINSKY L et al. O papel dos fatores genéticos na otite média. Medicina, Ribeirão Preto, 32: 57-64, jan./mar. 1999.

RESUMO: É sabido que a otite média aguda pode ser causada por fatores ambientais, como freqüentar creches, fumo passivo, curto período de amamentação e baixas condições sócio-econômicas. A revisão das pesquisas recentes, contudo, sugere que fatores genéticos também contribuem de forma significativa para a ocorrência da otite média aguda, recorrente e da otite média crônica, com efusão. Embora não existam estudos genéticos específicos, há consistentes evidências em favor da transmissão genética de uma suscetibilidade para otite média. A história familiar, características raciais, a freqüência de antígenos HLA e de marcadores genéticos, entre outros fatores, são algumas das evidências que serão apresentadas nesta revisão de literatura.

UNITERMos: Otite Média. Marcadores Genéticos. Sexo. Raças. Genética. Anormalidades. Imunologia.

\section{INTRODUÇÃO}

A otite média aguda (OMA) é um processo inflamatório da mucosa do ouvido médio que pode estender-se a outros espaços aéreos do osso temporal, pela própria continuidade de seu revestimento mucoso. A otite média é uma das causas mais freqüentes de visitas ao pediatra. Até os três (3) anos, mais de $80 \%$ das crianças tiveram, pelo menos, um episódio de OMA com efusão e $46 \%$ tiveram, pelo menos, três destes episódios.

A otite média é uma doença multifatorial e não completamente compreendida, envolvendo fatores extrínsecos e intrínsecos. Dentre os fatores extrínsecos, freqüentar creche parece ser o fator mais associado com otites de repetição $0^{(1 / 4)}$.

Como todas as infecções respiratórias, a otite média ocorre de duas (2) a quatro (4) vezes mais freqüentemente no inverno e outono, coincidindo com a época de maior incidência de infecções respiratórias, virais $^{(5,6,7)}$. Além disso, a natação em piscinas aquecidas é importante fator de infecções de vias aéreas superiores (IVAS) ${ }^{(8)}$.

Outras circunstâncias que podem influenciar na incidência de OMA incluem tabagismo passivo ${ }^{(2)}$, morar em apartamento ao invés de casa ${ }^{(3)}$, morar em habitações inadequadas $^{(4)}$ e curto período de amamentação ${ }^{(9)}$. A significância destes fatores, no entanto, ainda está sob discussão( ${ }^{(10)}$.

Dentre os fatores intrínsecos, a idade é fator de destaque. A otite média aguda de repetição (OMAr) é altamente prevalente na primeira infância, principalmente entre o primeiro $\left(1^{\circ}\right)$ e o segundo $\left(2^{\circ}\right)$ anos de idade $(60 \% \text { das crianças })^{(8)}$. A freqüência declina após os sete (7) anos. Quanto mais precoce o primeiro surto de otite média aguda, mais propensa será a criança para $\mathrm{OMAr}^{(11,12,13)}$. Há um predomínio no sexo masculino $^{(14)}$. 
Outros fatores intrínsecos são as disfunções imunológicas (pois a alergia induz um sistema mucociliar, ineficaz) $^{(8)}$; a intolerância à lactose; algumas imunodeficiências temporárias, como a IgA secretora; as discinesias ciliares primárias (embora com mais raridade) ${ }^{(15)}$; e as disfunções de tuba auditiva, imatura até por volta dos sete (7) anos, que aumentam a predisposição para OMAr em crianças portadoras de insuficiência velofaríngea e de palatosquise, tanto pela anatomia peculiar desta estrutura na infância (curta, horizontalizada e ampla), quanto pela disfunção da musculatura velofaríngea ${ }^{(8,16)}$.

As hiperplasias das tonsilas faríngeas e palatina produzem disfunção velofaríngea e, eventualmente, obstrução do óstio tubárico, facilitando as otites médias por obstrução e refluxo. As adenoidites agudas de repetição também são causas freqüentes de otites médias ${ }^{(8,17)}$. O refluxo gastroesofágico deve ser suspeitado como causa de otites médias agudas, não acompanhadas de IVAS no lactente ${ }^{(18)}$.

Além de todos estes fatores, a genética parece ter um papel importante no desencadeamento da otite média aguda. Embora ainda não estejam totalmente compreendidos, fatores, como predisposição racial e familiar para OMA, têm sido avaliados por diversos estudos que enfatizam a importância da determinação da predisposição para o controle e a prevenção da doença, principalmente de suas seqüelas mais graves. O objetivo do presente trabalho é revisar as evidências apresentadas por estudos que discutem a influência do sexo, raça, predisposição familiar e anormalidades imunológicas na suscetibilidade à otite média.

\section{SEXO}

Indivíduos do sexo masculino têm mais episódios de otite média e se submetem a mais miringotomias e timpanoplastias do que indivíduos do sexo fe$\operatorname{minino}^{(8,11,19,20,21)}$.

Um estudo prospectivo e controlado revelou que, no grupo com otites de repetição, $61 \%$ dos indivíduos pertenciam ao sexo masculino e apenas 39\% ao sexo feminino, demonstrando uma relação de $2: 1^{(22)}$. Nesse mesmo estudo, observou-se que pais e irmãos de indivíduos no grupo com otites de repetição tiveram mais episódios de otite na infância, quando comparados aos pais e irmãos de indivíduos do grupo controle $(p<0,001)$. Esta diferença não foi observada em relação às mães ${ }^{(22)}$.

\section{RAÇA}

A influência de fatores raciais na otite média é evidenciada pelos achados de alguns estudos que detectaram uma maior frequiência de OMA em determinadas populações étnicas. A maior incidência relatada de otite média é entre índios apaches e navajos ${ }^{(16,23)}$, nos Estados Unidos.

As crianças apaches, adotadas por famílias não apaches tiveram incidência de otite similar às crianças em reservas indígenas ${ }^{(24)}$. Em determinado estudo com índios apaches, demonstrou-se que há uma maior facilidade de insuflação da trompa nesse grupo do que em caucasianos, o que resultaria em menor proteção ao ouvido médio no grupo índio ${ }^{(25)}$. OMA tem sido também constatada com maior frequiência em esquimós do Alasca e em crianças hispânicas do que em americanos caucasianos ${ }^{(26)}$. A freqüência é menor entre negros norte-americanos e africanos ${ }^{(27,28)}$. Em duzentas e vinte (220) crianças da Ilha da Páscoa, no Chile, nenhum caso de otite média foi identificado entre crianças nativas geneticamente puras (de origem étnica polinésia); a prevalência foi de $10 \%$ entre crianças nativas racialmente mistas (primeira geração, com um dos pais sendo nativo e outro nascido fora da Ilha) e de $11 \%$ entre crianças nascidas fora da Ilha ${ }^{(29)}$. Além disso, três crianças nativas, geneticamente impuras, descendentes da mesma família européia, apresentaram OMA. Essas crianças não eram irmãos nem primos em primeiro grau.

\section{PREDISPOSIÇÃO FAMILIAR}

Vários autores têm levantado evidências de que crianças com irmãos ou um dos pais com histórico de OMAr parecem estar em risco maior para contrair a doença ${ }^{(18,30,31,32)}$. Alguns estudos sugerem que o risco de contrair OMAr e otite média crônica, com efusão (OMCE) é de 1,6 a 4,2 vezes maior entre crianças com um irmão afetado do que entre crianças sem irmão afetado ${ }^{(11,30,31,33,34,35)}$. Estudos prospectivos, utilizando análise multivariada, verificaram que o relato de um irmão afetado é fator de risco independente para OMAr ou para OMCE ${ }^{(11,31,35)}$. Marchant et al..$^{(36)}$ verificaram que $80 \%$ das crianças com OMCE também tiveram OMAr durante o primeiro ano de vida. Em contrapartida, Schwartz et al. verificaram que $45 \%$ das crianças com história de OMAr desenvolveram OMCE, durante seis (6) meses de acompanhamento ${ }^{(37)}$. 
Em um estudo de coorte não selecionada, com mil, trezentas e seis (1306) crianças suecas, acompanhadas desde o nascimento até sete (7) anos de idade, avaliou-se o impacto de fatores familiares na incidência de OMS cujo tratamento necessitasse de tubo de ventilação. A incidência cumulativa de OMS foi quatro (4) vezes maior entre as crianças que tinham irmãos que também receberam esse tipo de tratamento para OMS ( $\mathrm{p}=0.0000)$. Nesse estudo, não houve diferença significativa entre meninos (4\%) e meninas (7\%) que tiveram inseridos tubos de ventilação para tratamento de OMS $(\mathrm{p}=0.10)^{(35)}$.

Rockley \& Evans ${ }^{(18)}$ realizaram um estudo no qual examinaram o ouvido de cento e quarenta e seis (146) pais e mães de setenta e três (73) crianças entre um (1) e quatro (4) anos de idade, com indicação de tratamento cirúrgico para OMS (miringotomia); também foram examinados setenta (70) pais e mães (controles) de trinta e cinco (35) crianças sem OMS. $\mathrm{O}$ estudo constatou a presença de anormalidades de membrana timpânica, sugerindo OMS prévia, em 39\% dos pais e mães de pacientes com OMS e em 14\% dos pais e mães de crianças controles ( $\mathrm{p}<0.001)$. Sessenta e seis por cento das crianças com OMS persistente tinham um ou ambos os pais apresentando anormalidades de membrana timpânica, em comparação com $26 \%$ dos controles $(\mathrm{p}<0.001)$. Da mesma forma, para $74 \%$ das crianças do grupo controle, nenhum dos pais apresentava anormalidades de membrana timpânica, enquanto que, no grupo com OMS, apenas $34 \%$ tinham ambos os pais sem anormalidades $(\mathrm{p}<0.001)^{(18)}$. As anormalidades são consideradas seqüelas de OMS na infância ${ }^{(38 / 41)}$. Em acompanhamentos de longa duração, com crianças que trataram OMS, mesmo após vários anos de resolução da efusão, a maioria dos pacientes (60-80\%) ainda mostra anormalidades na membrana timpânica ${ }^{(42 / 46)}$.

Em Mineápolis, nos Estados Unidos, Daly et al. $^{(47)}$ avaliaram trezentas e dezesseis (316) famílias de crianças com tubo de ventilação devido a OMAr ou OMCE. As famílias estudadas foram selecionadas a partir de crianças incluídas em estudos anteriores, portadoras de OMAr/OMCE, na época. Os membros da família, incluídos naquela investigação, eram parentes em primeiro grau dos pacientes selecionados, ou seja, pais, irmãos e filhos. Relataram história de OMAr/OMCE 23\% dos pais, $43 \%$ dos irmãos, $100 \%$ dos selecionados e $62 \%$ dos descendentes (filhos). Outros parâmetros avaliados foram exame otomicroscópico, mostrando evidência de OMAr/OMCE pré- via, que foi positivamente verificada em $21 \%$ dos pais, $33 \%$ dos irmãos, $84 \%$ dos selecionados e $46 \%$ dos filhos, além de timpanometria alterada, compatível com OMAr/OMCE, verificada em 35\%, 59\%, $80 \%$ e $62 \%$ dos pesquisados, respectivamente. Eram consideradas afetadas aquelas pessoas para quem dois dos três critérios utilizados fossem positivos (relato da história pelo próprio paciente ou por seus pais, exame otomicroscópico e timpanometria de multifreqüência). Dessa forma, $62 \%$ dos filhos e $32 \%$ dos irmãos foram classificados como afetados (OMAr/OMCE). A incidência de alteração de membrana timpânica compatível com OMAr/OMCE em irmãos dos pacientes, foi comparada com uma amostra da população, de doze (12) a dezessete (17) anos de idade, de 1966 a 1970. Os resultados mostraram alterações em $33 \%$ de todos os irmãos do estudo e em 19\% dos pais e irmãos nascidos no mesmo período que os controles, contra apenas 7\% de alterações nos controles. Os resultados indicam um risco aumentado em cinco (5) vezes para irmãos de pacientes com OMAr/OMCE e em 2,7 vezes para pais e irmãos controlados por coorte de nascimento. Isso confirma um alto índice de $\mathrm{OM}$ em famílias com um membro afetado em comparação com a população em geral $^{(47)}$.

A evidência de história de $\mathrm{OM}$ em duas ou mais gerações sugere fortemente a influência de fatores genéticos, pois, diferente da coincidência entre irmãos (que têm o mesmo ambiente de desenvolvimento), pais e seus descendentes têm influências ambientais diferentes durante a infância - período de maior risco para OM.

\section{ANORMALIDADES IMUNOLÓGICAS}

\subsection{Antígenos do Linfócito Humano (HLA)}

Está bem estabelecida a relação entre a freqüência de certos antígenos HLA e diversas entidades patológicas. Considerando a maioria das doenças associadas com antígenos HLA, o risco de morbidade em associação com a presença de um antígeno é relativamente moderado, sugerindo, assim, que um efeito direto do antígeno, no desenvolvimento de doença não pode ser predito ${ }^{(47,48)}$.

Apesar da baixa correlação entre a ocorrência de doenças infecciosas e antígeno HLA, existe uma relação entre a ocorrência de deficiência de $\operatorname{IgA}$ e a presença de certos antígenos HLA.

Em 1991, Kalm et al. ${ }^{(48)}$ investigaram a frequiência de HLA em quarenta e cinco (45) pacientes com 
OMA recorrente e compararam essa freqüência com o grupo controle. O antígeno HLA-A2 ocorreu em $80 \%$ (36/45) dos pacientes com OMA recorrente, contra $56 \%$ nos controles $(\mathrm{p}<0,001)$. Do subgrupo de onze (11) crianças com OMA recorrente, $10(91 \%)$ foram HLA-A3 positivo. O antígeno HLA-A3 ocorreu em somente $11 \%$ (5/45) do grupo com OMA recorrente, em comparação com $28 \%$ dos controles $(\mathrm{p}<0,005)$. Entre as vinte e duas (22) crianças acompanhadas prospectivamente, sem episódios de OMA durante os três (3) primeiros anos de vida, a freqüência de HLA-A2 foi de $45 \%$ (10/22) e HLA-A3 foi $32 \%$ (7/22). Os resultados indicaram a existência de uma relação entre OMA recorrente e locus HLA-A2, sugerindo o envolvimento de fatores genéticos ${ }^{(48)}$.

Em outro estudo, Daly et al. investigaram as freqüências dos mesmos trinta e quatro (34) antígenos HLA em quarenta (40) crianças que tinham sido regularmente acompanhadas, durante, pelo menos, seis (6) anos, por otite média secretora crônica. HLA-A2 foi encontrado em 52\% e HLA-A3 em 27,5\% dessas crianças. A freqüência de HLA-A2 foi significativamente menor em pacientes com OMS crônica do que em crianças com OMA recorrente. Os resultados indicam uma diferença da influência genética na patogênese de OMA recorrente e de OMS crônica ${ }^{(47)}$.

\subsection{Citocinas}

Durante episódios de otite média crônica secretora, a retenção de mediadores inflamatórios e células, no ouvido médio, determina a persistência da inflamação, com as alterações patológicas e auditivas daí decorrentes ${ }^{(2,49)}$. Citocinas são glicoproteínas produzidas por macrófagos e outras células. As atividades das citocinas incluem produção de febre, osteoclastos, fibroblastos, fagócitos, ativação de células citotóxicas e inibição de crescimento de cartilagem, ossos e células endoteliais ${ }^{(49)}$.

Yellon et al. $(1991)^{(50)}$ demonstraram, nas secreções do ouvido médio de portadores de otite secretora crônica, a presença de significativas quantidades de citocinas, do tipo interleucina-1, interleucina-2, fator de necrose tumoral alfa e gama interferon, além de ausência do fator estimulante de granulócitos e macrófagos e interleucina-4. As propriedades ativadoras de ação citotóxica, osteoclástica e fibroblástica podem ser responsáveis pela lesão da mucosa, erosão óssea e fibrose e, deste modo, pela surdez que se observa em alguns casos de otite média secretora crônica ${ }^{(51)}$. As evidências indicam que a função diferenciada dos macrófagos tem um possível fator de controle central, na persistência da efusão do ouvido médio. Estudos sugerem que os altos índices de fator alfa de necrose tumoral estão relacionados com a persistência da otite secretora ${ }^{(8,52,53)}$.

A retenção de produtos inflamatórios no espaço do ouvido médio, durante infecção e disfunção da trompa de Eustáquio, com drenagem pobre, pode resultar em progressivo estado inflamatório crônico, que tem o potencial de gerar lesões permanentes de mucosa, fibrose, erosão óssea e surdez. A participação, neste processo, de células mediadas pelo processo inflamatório, pode contribuir de forma importante para a otite média secretora crônica, como parece sugerir a presença de neutrófilos, linfócitos, macrófagos e remanescentes celulares dos fluidos da efusão, do espaço subepitelial e do espaço do ouvido médio. Contribuições adicionais de anticorpo, complemento, coagulação, fibrinólises, prostaglandinas e outros sistemas, têm sido identificadas na otite média com efusão ${ }^{(51)}$.

Maxwell et al. (1994) ${ }^{(54)}$ demonstraram que a interleucina- 8 , juntamente com a interleucina- 1 beta e o fator de necrose tumoral beta, são responsáveis pelo acúmulo de leucócitos no espaço do ouvido médio e pela ativação desses leucócitos, com subseqüente lesão do tecido. Esse estudo proporcionou suporte à idéia de que a interleucina-1 beta, o fator de necrose tumoral beta e a interleucina-8 estão intimamente envolvidos na cascata inflamatória do ouvido médio, sugerindo que a regulação dessas citocinas tem um papel promissor no futuro terapêutico da otite média secretora com efusão.

Está demonstrado que a interleucina- 8 tem uma potente e seletiva atividade quimiotáctica para neutrófilos. Sabe-se, também, que a interleucina- 8 está envolvida em várias doenças, como a psoríase, a artrite reumatóide e a fibrose pulmonar idiopáti$\mathrm{ca}^{(49,55,56)}$. Takeuchi et al. (1994) ${ }^{(49)}$ demonstraram a existência de uma expressão genética da interleucina-8 em efusões do ouvido médio, tanto de adultos como de crianças.

\subsection{Alterações da Imunidade}

Sobre esse aspecto, é importante citar os resultados de Prellner et al. $(1985)^{(10)}$ :

- Fatores de imunidade tumoral (IgG, IgA, IgM) foram semelhantes entre crianças com e sem OMA recorrente. 
- Pacientes com OMA recorrente tiveram níveis menores de anticorpos específicos contra pneumococo tipo 6A e 19F. Essa diferença imunológica parece já existir desde o nascimento.

- Há uma baixa concentração de anticorpos contra o hemófilo influenza não tipável, em indivíduos com OMA recorrente.

- Aberrações do primeiro fator de complemento (C1) parecem ter importância limitada como fator predisponente para o desenvolvimento da OMA recorrente.

Outro estudo $^{(57)}$ analisou os marcadores G1m(1), $\operatorname{Glm}(2), \operatorname{G1m}(3)$ e $\mathrm{Km}(1)$ em vinte (20) famílias, compreendendo um dos pais e a criança com história de OMA recorrente e um dos pais sem história de OMA recorrente. Além disso, G2m(23) foi identificado em quarenta e sete (47) crianças sem OMA. A distribuição de marcadores Gm e Km entre OMA recorrente e controles não revelou diferença significativa. Crian- ças com OMA recorrente tiveram freqüência de G2m(23), enquanto outras observações em adultos demonstram baixa resposta ao antígeno polissacarídeo, preferencialmente $\mathrm{G} 2 \mathrm{~m}(23)$. Os resultados indicam que os mecanismos responsáveis por baixa concentração de anticorpos antipolissacarídeos pneumocócicos, em crianças com OMA recorrente, podem diferir dos mecanismos que fazem com que adultos respondam, quando expostos a polissacarídeos pneumocócicos.

\section{DISCUSSÃO E CONCLUSÃO}

O conhecimento de fatores genéticos, envolvidos na ocorrência de OMA, auxilia na determinação de grupos de crianças com maior risco, particularmente para otites voltadas a uma cronificação (Tabela I). Isso possibilita a tomada de medidas de prevenção e tratamento precoce mais efetivos.

Tabela I - Principais fatores de predisposição genética à otite média aguda, conforme revisão da literatura*

\begin{tabular}{ll} 
Fator predisponente & Descrição \\
\hline Sexo & Ocorre mais entre o sexo masculino \\
Raça & $\begin{array}{l}\text { A relação de risco por raça é esquimós e hispânicos > auca- } \\
\text { sianos > negros }\end{array}$ \\
$\begin{array}{l}\text { Membro da família com his- } \\
\text { tórico de OMA }\end{array}$ & $\begin{array}{l}\text { Risco maior para crianças com membro da família (pais, } \\
\text { irmão) afetado ou com anormalidades da membrana tim- } \\
\text { pânica, sugerindo OMS prévia }\end{array}$
\end{tabular}

Referência

Anormalidades Imunológicas

- HLA

- Citocinas

- Alterações da imunidade
HLA-A2 e A3 ocorrem com freqüência significativamente maior em pacientes com OMA recorrente. Pode haver uma relação entre OMA recorrente e HLA-A2 local, sugerindo o envolvimento de fatores genéticos.

Constatou-se uma freqüência de HLA-A2 significativamente menor em pacientes com OMS crônica do que em crianças com OMA recorrente. Os resultados indicam uma diferença da influência hereditária na patogênese de OMA recorrente e de OMS crônica.

Constatou-se expressão genética da interleucina-8 em efusões de ouvido médio em crianças e adultos.

A regulação da interleucina- 1 beta, fator de necrose tumoral beta e interleucina-8, pode ter um papel importante no futuro terapêutico da otite média secretora com efusão.

Pacientes com OMA têm níveis menores de anticorpos contra pneumococo 6A e 19F; em OMA recorrente, pacientes apresentam baixa concentração de anticorpos contra hemófilo influenza não tipável; aberrações do primeiro fator de complemento $\mathrm{C} 1$ pode ter importância como fator predisponente para OMA recorrente.
$8,11,19-21$

23-29

11,18, 30, 31, 33-35, 47

47,48

$2,4,49,51,54$

10

${ }^{\star} \mathrm{OMA}=$ otite média aguda; OMS = otite média secretora. 
Especial atenção deve ser dada às crianças com $\mathrm{OM}$ em idade precoce (menos de seis (6) meses) ou cujos pais ou irmãos apresentam histórico de otite. Esses pacientes têm chances maiores de apresentar OMAr ou OMCE devido à predisposição genética. Identificando-se tais pacientes, deve-se tratá-los precocemente e mantê-los sob acompanhamento regular de intervalos curtos, a fim de evitar a cronificação da otite e a grande morbidade associada a tal condição. As crianças com OMAr ou com OMC possuem alto risco para o desenvolvimento de seqüelas, inclusive perda auditiva, efeitos deletérios do ouvido médio e de suas estruturas (timpanosclerose, otite adesiva, perfuração permanente da membrana timpânica...) e déficit de aprendizagem, com dramáticas repercussões para o futuro dessas crianças. O tratamento precoce e adequado da otite aguda, assim como o controle de seus fatores predisponentes, poderá resultar em uma menor prevalência de otite média crônica.

Igualmente, nos grupos predispostos, torna-se necessário o estudo de anormalidades imunológicas ou relacionadas com mediadores inflamatórios, para que, com a adequada conduta, possamos evitar as consequiências antes referidas. Posteriores estudos genéticos fornecerão, provavelmente, contribuições sobre a compreensão da transmissão genética da $\mathrm{OM}$ e poderão, futuramente, criar perspectivas para o controle da suscetibilidade individual para OM.

LAVINSKY L et al. The role of genetic factors in otitis media. Medicina, Ribeirão Preto, 32: 57-64, jan./march 1999.

ABSTRACT: It is well-known that otitis media can be caused by environmental factors, such as attending day care centers, passive smoking, early interruption of breast feeding, and unfavorable socio-economic conditions. The review of current literature, however, suggests that genetic factors also contribute significantly to the occurrence of recurrent acute otitis media and otitis media with effusion. Although there are no specific genetic studies, there is consistent evidence to support the idea of genetically transmitted susceptibility to otitis media. Family history, racial characteristics, HLA and genetic marker frequency are some of the topics discussed in the literature, which will be presented in this review.

UNITERMS: Otitis Media. Genetic Markers. Sex. Racial Stocks. Genetics. Immunology Abnormalities.

\section{REFERÊNCIAS BIBLIOGRÁFICAS}

1 - TOS M; POULSEN G \& BORCH J. Tympanometry in 2-year-old children. Otorhinolaryngology 40: 77-85, 1978.

2 - STÄHLBERG MB; RUUSKANEN O \& VIROLAINEN E. Risk factors for recurrent otitis media. Pediatr Infect Dis 5: 30-32, 1986.

3 - LUNDGREN K; INGVARSSON L \& OLOFSSON B. Epidemiologic aspects in children with recurrent acute otitis media. In: LIM DJ et al., eds. Recent advances in otitis media with effusion, BC Decker, Philadelphia, p. 22, 1984.

4 - PUKANDER $\mathrm{J}$ et al. Risk factors affecting the occurrence of acute otitis media among 2-3-year-old urban children. Acta Otolaryngol (Stockh) 100: 260-265, 1985.

5 - BIRCH L et al. A prospective epidemiological investigation of secretory otitis media and tubal dysfunction in children attending day-care centres. RBM-Otorrinolaringol 46:210-216, 1984.

6 - BYLANDER A. Upper respiratory tract infection and eustachian tube function in children. Acta Otolaryngol (Stockh) 97: 343-349, 1984.
7 - TOS M; POULSEN G \& BORCH J. Etiologic factors in secretory otitis. Arch Otolaryngol 105: 582-588, 1979.

8 - MAXSON S \& YAMAUCHI T. Acute otitis media. Pediatr Rev 17: 191-195, 1996.

9 - SIPILA M; PUKANDER J \& KARMA P. Incidence of acute otitis media up to the age of $1 \frac{1 / 2}{2}$ years in urban infants. Acta Otolaryngol (Stockh) 104: 138-145, 1987.

10 - PRELLNER K et al. Recurrent otitis media: genetic immunoglobulin markers in children and their parents. Int $\mathbf{J}$ Pediatr Otorhinolaryngol 9: 219-225, 1985.

11 - TEELE DW; KLEIN JO \& ROSNER B. Epidemiology of otitis media during the first seven years of life in children in greater Boston: a prospective, cohort study. J Infect Dis 160: 83-94, 1989.

12 - PRELLNER $\mathrm{K}$ et al. Pneumococcal serum antibody concentrations during the first three years of life: a study of otitis-prone and non-otitis-prone children. Int $\mathbf{J}$ Pediatr Otorhinolaryngol 17: 267-279, 1989.

13 - GOYCOOLEA MV. Otitis media: The pathogenesis approach. Otolaryngol Clin North Am 24: 787-794, 1991. 
14 - SIH T et al. Prophylaxis for recurrent acute otitis media: a Brazilian study. Int J Pediatr Otorhinolaryngol 25: 19-24, 1993.

15 - PEDERSEN M \& MYGIND N. Rhinitis, sinusitis and otitis media in Kartagener's syndrome (primary ciliary syskinesia). Clin Otolaryngol I 7: 373-380, 1982.

16 - BEERY QC et al. Eustachian tube function in an American Indian population. Ann Otol Rhinol Laryngol 89: 28-33, 1980. Suppl.

17 - SMYTH GDL \& HALL S. Aetiology and treatment of persistent middle ear effusion. J Laryngol Otol 97: 1085-1089, 1983.

18 - ROCKLEY TJ \& EVANS PHR. Secretory otitis media - Evidence for an inherited aetiology. J Laryngol Otol 100: 389-393, 1986.

19 - KERO P \& PIEKKALA P. Factors affecting the occurrence of acute otitis media during the first year of life. Acta Paediatr Scand 76: 618-623, 1987.

20 - INGVARSSON L; LUNDGREN K \& OLOFSSON B. Incidence and risk factors of acute otitis media in children: Longitudinal cohort studies in an urban population. In: LIM DJ, ed. Recent advances in otitis media, BC Decker, Philadelphia, p. 6-8, 1988.

21 - ALHO OP et al. Is a child's history of acute otitis media and respiratory infection already determined in the antenatal and perinatal period? Int J Pediatr Otorhinolaryngol 19: 129-137, 1990.

22 - STENSTRÖM C \& INGVARSSON L. Otitis-prone children and controls: a study of possible predisposing factors. 1. Heredity, family background and perinatal period. Acta Otolaryngol (Stockh) 117: 87-93, 1997.

23 - WIET RJ. Patterns of ear disease in the southwestern American Indian. Arch Otolaryngol Head Neck Surg 105: 381-385, 1979.

24 - SPIVEY G \& HIRSCHHORN N. A migrant study of adopted Apache children. Johns Hopkins Med J 40: 43-46, 1977.

25 - BLUESTONE CD \& KLEIN JO. Otitis media in infants and children. W.B. Saunders, Philadelphia, 1988. 310 p.

26 - GIEBINK GS. Epidemiology and natural history of otitis media. In: LIM DS et al., eds. Recent advances in otitis media with effusion, BC Decker, Philadelphia, p. 5-9, 1984.

27 - GRIFFITH T. Epidemiology of otitis media: an interracial study. Laryngoscope 82: 22-30, 1979.

28 - MILLER AS et al. A point prevalence of otitis media in a Nigerian village. J Pediatr Otorhinolaryngol 5: 19-29, 1983.

29 - GOYCOOLEA HG; GOYCOOLEA MV \& FARFAN CR. Racial and familial factors in otitis media. Arch Otolaryngol Head Neck Surg 114: 147-149, 1988.

30 - HARSTEN G et al. Recurrent acute otitis media: a prospective study during the first three years of life. Acta Otolaryngol (Stockh) 107: 111-119, 1989.
31 - SIPILA M et al. The Bayesian approach to the evaluation of risk factors in acute and recurrent acute otitis media. Acta Otolaryngol (Stockh) 106: 94-101, 1988.

32 - TEELE DW; KLEIN JO \& ROSNER BA. Epidemiology of otitis media in children. Ann Otol Rhinol Laryngol 89: 5-6, 1980. Suppl.

33 - KRAEMER MJ et al. Risk factors for persistent middle ear effusions. Otitis media, catarrh, cigarette smoke exposure and atopy. JAMA 249: 1022-1025, 1983.

34 - BLACK N. The aetiology of glue-ear - a case-control study. Int J Pediatr Otorhinolaryngol 9: 121-133, 1985.

35 - RASMUSSEN F. Protracted secretory otitis media. The impact of familial factors and day-care center attendance. Int $\mathbf{J}$ Pediatr Otorhinolaryngol 26: 29-37, 1993.

36 - MARCHANT CD et al. Course and outcome of otitis media in early infancy: a prospective study. J Pediatr 104: 826-831, 1984.

37 - SCHWARTZ RH; RODRIGUEZ WJ \& GRUNDFAST KM. Duration of middle ear effusion after acute otitis media. Pediatr Infect Dis J 3: 204-207, 1984.

38 - SADÉ J. Secretory otitis media and its sequelae. In: SADE J, ed. Monographs in clinical otolaryngology, Churchill-Livingstone, New York, v. 1, p. 89-101, 1979.

39 - DAWES JDK. The aetiology and sequelae of exudative otitis media. J Laryngol Otol 84: 583-610, 1970.

40 - SMYTH GDL. Middle ear effusions: a consideration of the factors involved in their aetiology, maintenance and treatment. Clin Otolaryngol 3: 479-490, 1978.

41 - TOS M et al. Spontaneous course of secretory otitis media and changes in the eardrum. Arch Otolaryngol 110: 281-289, 1984.

42 - BEN-AMI $M$ et al. Otitis media with effusion - complications after treatment. J Laryngol Otol 97: 1091-1093, 1983.

43 - TOS M \& POULSEN G. Secretory otitis media, late results of treatment. Arch Otolaryngol 102: 672-675, 1976.

44 - MAWSON SR \& FAGAN P. Tympanic effusions in children: long-term results of treatment by myringotomy, aspiration and indwelling tubes. J Laryngol Otol 86: 105-118, 1972.

45 - BARFOED A \& ROSBORG J. Secretory otitis media: long term observations after treatment with grommets. Arch Otolaryngol 106: 553-556, 1980.

46 - HAUGETO OK et al. Chronic secretory otitis media. Acta Otolaryngol (Stockh) 360: 192-194, 1979. Suppl.

47 - DALY KA et al. The family study of otitis media: design and disease and risk factor profiles. Genet Epidemiol 13: 451-468, 1996.

48 - KALM O; JOHNSON U \& PRELLNER K. HLA frequency in patients with chronic secretory otitis media. Int $\mathbf{J}$ Pediatr Otorhinolaryngol 30: 151-157, 1994. 
49 - TAKEUCHI K et al. Interleukin-8 gene expression in middle ear effusions. Ann Otol Rhinol Laryngol 103: 404-407, 1994.

50 - YELLON RF et al. Characterization of cytokines present in middle ear effusions. Laryngoscope 101: 165-169, 1991.

51 - SAKAKURA $\mathrm{K}$ et al. Endotoxin and lysosomal protease activity in acute and chronic otitis media with effusion. Ann Otol Rhinol Laryngol 99: 379-385; 1990.

52 - LEONARD EJ \& YOSHIMURA T. Neutrophil attractant/activation protein 1 (Nap-1[interleukin 8]). Am J Respir Cell Mol Biol 2: 479-486, 1990.

53 - BAGGIOLINI M; WALZ A \& KUNKEL SL. Neutrophil-activating peptide $1 /$ interleukin-8, a novel cytokine that activates neutrophils. J Clin Invest 84: 1045-1049, 1989.
54 - MAXWELL KS et al. Interleukin-8 expression in otitis media. Laryngoscope 104: 989-995, 1994.

55 - SCHRÖDER JM \& CHRISTOPHERS E. Identification of C5ades arg and anionic neutrophil-activating peptide (ANAP) in psoriatic scales. J Invest Dermatol 87: 53-58, 1986.

56 - WATSON ML et al. Elevation of PMN cytosolic free calcium and locomotion stimulated by novel peptides from IL-1 treated human synovial cell cultures. Biochem Biophys Res Commun 155: 1154-1160, 1988.

57 - KALM O et al. HLA frequency in patients with recurrent acute otitis media. Arch Otolaryngol Head Neck Surg 117: 1296-1299, 1991.

Recebido para publicação em 21/09/98

Aprovado para publicação em 08/12/98 